\title{
Grating interferometer based scanning setup for hard x-ray phase contrast imaging
}

\author{
C. Kottler, ${ }^{\text {a) }}$ F. Pfeiffer, O. Bunk, C. Grünzweig, and C. David \\ Paul Scherrer Institut, CH-5232 Villigen PSI, Switzerland
}

(Received 31 January 2007; accepted 12 March 2007; published online 30 April 2007)

\begin{abstract}
In x-ray radiography, particularly for technical and industrial applications, a scanning setup is very often favorable when compared to a direct two-dimensional image acquisition. Here, we report on an efficient scanning method for grating based x-ray phase contrast imaging with tube based sources. It uses multiple line detectors for staggered acquisition of the individual phase-stepping images. We find that the total exposure time does not exceed the time needed in an equivalent scanning setup for absorption radiography. Therefore, we conclude that it should be possible to implement the method into a scanning system without affecting the scanning speed or significant increase in cost but with the advantage of providing both the phase contrast and the absorption information at once. (C) 2007 American Institute of Physics. [DOI: 10.1063/1.2723064]
\end{abstract}

\section{INTRODUCTION}

Phase sensitive $\mathrm{x}$-ray techniques have become an attractive alternative method for imaging applications because they use the phase shift rather than the absorption as the imaging signal and thus they can deliver additional and complementary information on the sample. In the past years a variety of such techniques has been developed and many applications have been reported. ${ }^{1-17}$ However, not one of these techniques has become so far an instrument to be routinely used in commercial applications such as in medical imaging, security screening, nondestructive testing or quality inspection of commercial products. Because of the need for highly temporal and/or spatial coherent radiation sources these techniques cannot be applied in combination with conventional high power and broadband x-ray tubes which would be essential for routine applications.

Recently we have developed an $\mathrm{x}$-ray technique providing phase sensitive $\mathrm{x}$-ray images with conventional broadband X-ray tube sources. ${ }^{18-20}$ This technique is based on transmission gratings that are placed in the X-ray beam (Fig. 1). The phase grating $G_{1}$ and the absorption grating $G_{2}$ form the phase sensitive interferometer. They are located between the sample and the detector. Due to recent advances in the micro-fabrication processes ${ }^{21,22}$ large area gratings for large fields of view are now available. Thus, phase contrast images can be achieved of centimeter sized samples. Moreover, by taking advantage of the so-called phase-stepping data acquisition mode, ${ }^{23-25}$ this technique provides not only the advantage of phase sensitive imaging but also the complementary conventional absorption radiography information at the same time. Such a setup with only two gratings requires a high degree of spatial coherence. This means, that the method would only work with very small micro focus sources placed at a large distance, resulting in low flux densities and thus long exposure times. To overcome this severe limitation, we

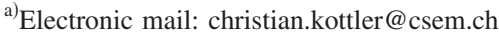

use a third grating $G_{0}$ mounted close to the x-ray source, dividing the extended incident beam into an array of sources with sufficient spatial coherence. If the grating periods fulfill the condition $p_{0}=(l / d) p_{2}$ (see Fig. 2), each of the individual sources contributes constructively to the image formation. In consequence, large and incoherent $\mathrm{x}$-ray sources can be applied. These gratings can be considered as a rather simple add-on to any existing $x$-ray imaging setup. Therefore, this technique has the potential to provide phase sensitive imaging at high power and broadband $\mathrm{x}$-ray tubes for any kind of application.

As diverse as the applications of $\mathrm{x}$-ray imaging, e.g., medicine, biology, pharmacy, quality inspection, nondestructive testing, and homeland security, are the needs for adequate instrumentation. Because some of them require fast, reliable, and cost-effective instrumentation, many of the existing absorption contrast systems have been realized as a scanning setup. Thereby, large area two-dimensional detectors can be omitted and inexpensive line detectors can be used instead. Furthermore, scanning-type systems can be easily adopted if the objects to be screened come one after the other as is the case for products coming on a conveyor belt. Furthermore for very large samples scanning is very often the only practicable approach.

Running the grating interferometer in the scanning mode (detector and grating lines in parallel arrangement) not only provides the benefits of line detectors but also solves some of the problems when large area gratings are used, i.e., alignment, homogeneity of fabrication or nonorthogonal angle of incidence. However, with a single line detector the phasestepping procedure becomes very slow because in every step of the sample scan as many exposures have to be taken and read out as phase-steps are desired for the analysis. In this work a scanning method is presented that uses several parallel line detectors. The signals from the simultaneous exposures of the individual lines can be transformed into phase-stepping series and thus the latter can be acquired in a single shot. 


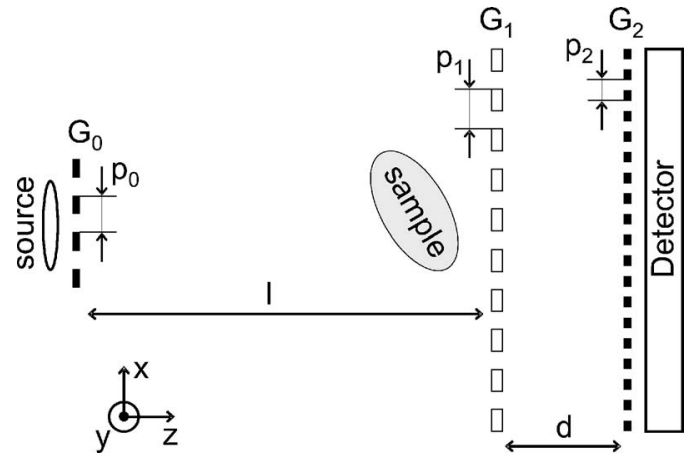

FIG. 1. Grating interferometer setup consisting of a $\mathrm{x}$-ray tube (Seifert ID3000, tungsten anode operated at $35 \mathrm{kV} / 30 \mathrm{~mA}$ ) and three gratings $G_{0}$, $G_{1}$, and $G_{2}$ with periods $p_{0}=73 \mu \mathrm{m}, p_{1}=3.89 \mu \mathrm{m}$, and $p_{2}=2.0 \mu \mathrm{m}$, respectively. The distances between the gratings were $l=168 \mathrm{~cm}$ and $d=4.4 \mathrm{~cm}$.

\section{EXPERIMENTAL SETUP}

\section{A. Grating interferometer setup}

Grating interferometry, fabrication and the x-ray tube setup have already been described in detail elsewhere. ${ }^{18-22,24-28}$ For our measurements reported here the parameters of the setup (as compiled in Fig. 1) were optimized for $28 \mathrm{keV}$ photon energy. Furthermore, our detector was one module of the single photon counting Medipix 2 detector. $^{29}$ It consists of a $700 \mu \mathrm{m}$ thick $\mathrm{Si}$ absorber layer and a CMOS readout chip underneath with $256 \times 256$ pixels of $55 \mu \mathrm{m}$ size each. Thus, the total image size was $14 \times 14 \mathrm{~mm}^{2}$. Multiple line detectors (with precisely defined distances between them) were achieved by selectively reading out individual columns of the Medipix2 module.

\section{B. Multiple line detector phase stepping scanning method}

Ideal alignment of the gratings $G_{1}$ and $G_{2}$ means that their lines are parallel to each other (along the $y$ axis, vertical direction) and that the effective periodicity of the microscopic interference pattern $\left[I(x)\right.$, see Fig. 2] generated by $G_{1}$ equals the periodicity of $G_{2}$. Misalignment of the gratings

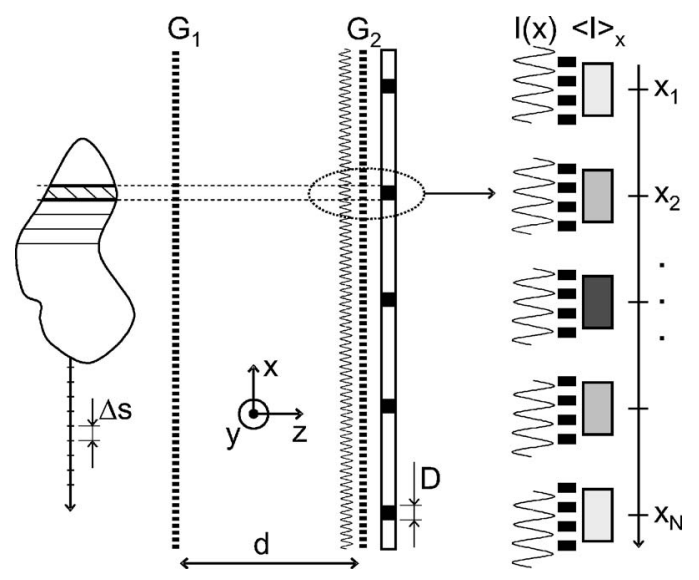

FIG. 2. Setup for scanning with the grating interferometer. Phase-stepping is achieved by slightly increasing the inter grating distance $d+\Delta d$ and using the signal from multiple line detectors in the appropriate sample scanning position.
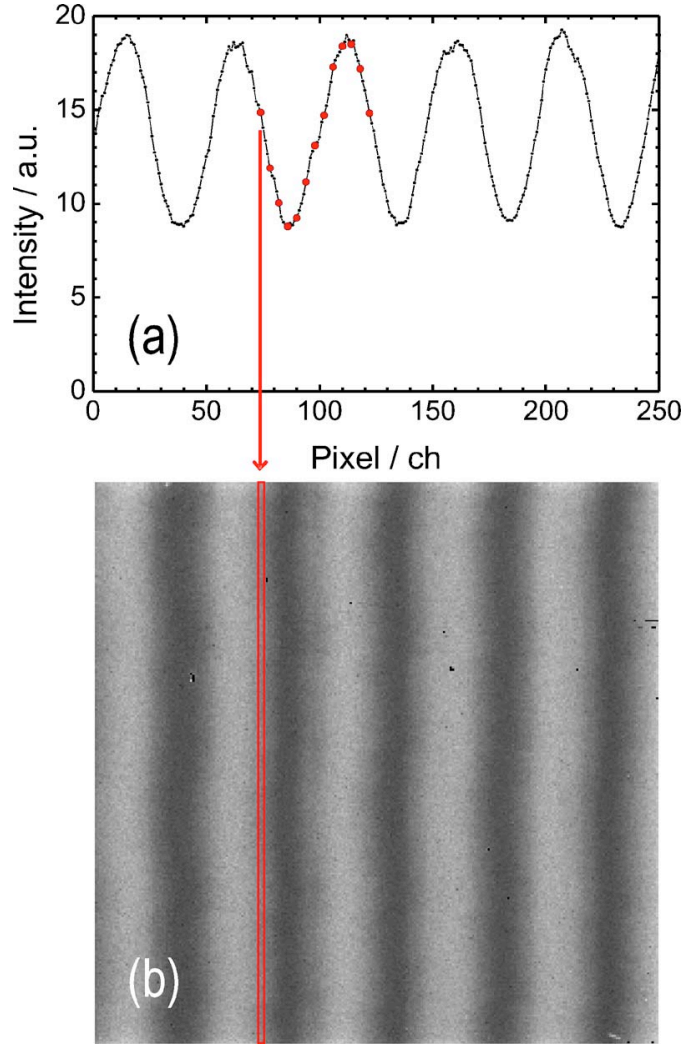

FIG. 3. (a) Intensity profile obtained from the projection of the image (b) along the indicated vertical axis. (b) Image acquired with the Medipix 2 detector containing vertical Moiré fringes that arose due to a mismatch in the inter grating distance of $\Delta d=2 \mathrm{~mm}$.

can easily be monitored as it gives rise to linear Moiré fringes. These Moiré fringes can be thought of as local varying shifts of the grating bars of $G_{1}$ with respect to those of $G_{2}$. For example, if the gratings are slightly distorted with respect to the $z$ axis (see Fig. 1) horizontal Moiré fringes arise (along the $x$ axis). Indeed, a slight mismatch of the inter grating distance $d^{\prime}=d+\Delta d$ causes vertical fringes (along the $y$ axis) because the beam divergence ${ }^{30}$ changes the effective periodicity of the microscopic interference pattern to $p_{2}^{\prime}=p_{2}+(\Delta d / l) p_{1}$ and thus $p_{2}^{\prime}$ differs from the $G_{2}$ periodicity $p_{2}$. Therefore, the intensity of the radiation transmitted through the gratings becomes a varying function of the $x$ coordinate depending on the relative position of the absorbing bars of $G_{2}$ with respect to the maxima of the microscopic interference pattern $I(x)$. In Fig. 3(b) an image recorded with the Medipix2 is shown where all parameters for the grating alignment were fully optimized, but the distance $d$ was increased by $\Delta d=2 \mathrm{~mm}$.

Figure 2 illustrates that, in the case of vertical Moiré fringes, equidistantly distributed individual lines of the detector $\left(x_{1}, x_{2}, \ldots, x_{N}\right)$ correspond to different relative position of $G_{1}$ and $G_{2}\left(\Delta x_{1}, \Delta x_{2}, \ldots, \Delta x_{N}\right)$. These relative position coordinates $\Delta x_{i}$ can be thought of as the coordinates of steps in a phase-stepping series. The idea for the scanning method is that the sample is moved in steps of $\Delta s$ in the horizontal direction and that the detector lines $x_{1}, x_{2}, \ldots, x_{N}$ are read out for each position. Then the image recorded with the line detector at $x_{i}$ and the indicated slice of the sample in front of 


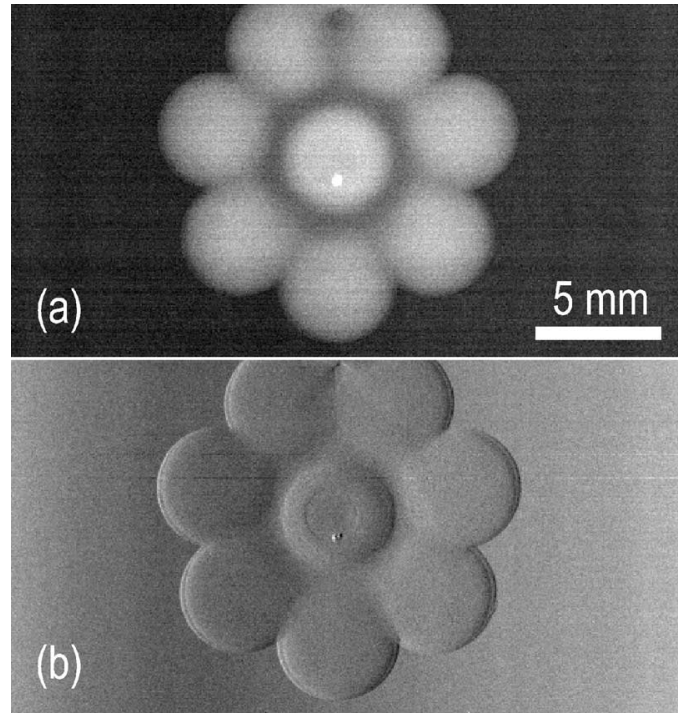

FIG. 4. (a) Absorption image and (b) differential phase contrast image acquired by scanning of a flowerlike test sample made from plastic. In total 840 scanning steps were performed yielding an image of $256 \times 840$ pixels.

it belongs to the $i$ th phase-step for the radiograph of the selected slice.

In this way, a series of $i=1, \ldots, N$ images is obtained, where each of them corresponds to a phase-stepping position $\Delta x_{i}$. From this data set the absorption and differential phase contrast image is achieved by using the phase-stepping analysis algorithm. ${ }^{23-25}$ The algorithm requires that the range over which the phase-stepping scan is carried out corresponds to integer multiples of one period. In terms of the scanning setup this means that the number of detector lines $N$ and the distance $D$ between them matches integer multiples of the distance between adjacent Moiré fringes. In Fig. 3(a) an example is shown where every fourth line and $N=12$ lines in total were read out from the Medipix2 detector, covering exactly one Moiré fringe.

Since the images taken for each phase-step position are recorded with different line detectors and at different positions of the gratings the measured signals also depend on the detector efficiency and the effectively transmitted x-ray intensity through the gratings. This overall efficiency can be determined for each pixel by acquiring a series of images with no sample in the beam and by stepping the grating $G_{2}$. The average of the intensity of each pixel over one period $p_{2}$ is proportional to the overall efficiency and thus can be used to normalize the data set of the sample measurement.

The sample scanning step size $\Delta s$ has to be slightly smaller than the distance $D$ between two detector lines to compensate for the beam divergence, i.e., $\Delta s=l /(l+d) D$, assuming that the sample is right in front of the grating $G_{1}$. The scanning translation stage must have sufficient precision for accurately positioning the sample in steps of $\Delta s$, which is $\approx 220 \mu \mathrm{m}$ in the present case.

\section{EXAMPLE AND APPLICATION}

Figure 4 shows a successful application example of the scanning method to a flowerlike test sample made from plastic $(\varnothing \approx 1.5 \mathrm{~cm})$. As indicated in Fig. 3, N=12 vertical lines (every fourth line) of the Medipix2 detector were read out in total and then reorganized as a phase stepping series. The absorption [Fig. 4(a)] and the differential phase contrast image [Fig. 4(b)] retrieved consist of $840 \times 256$ pixels each, whereby 840 represents the number of scanning steps performed. The exposure time was $5 \mathrm{~s}$ per scanning step. Thus, the total length of the scan in this example was $\approx 46 \mathrm{~mm}$ and the total acquisition time was $\approx 70 \mathrm{~min}$.

The number of lines and the spacing between them was arbitrarily chosen for this example. Only the condition that the selected sequence has to match exactly one Moiré fringe was taken into account. In a setup consisting of a given number of detector lines with fixed spacing this condition can be fulfilled by optimizing the inter grating distance $d$ such that the density of Moiré fringes appropriately matches the detector lines.

\section{CONCLUSION}

It was shown that by using several equidistant line detectors sample scanning can successfully be carried with a grating interferometer. This procedure simultaneously yields conventional absorption images and phase contrast images. The individual detector lines represent the sequence of a phase-stepping series. Since they can be acquired in one exposure, no extra exposure time is required if compared to a standard absorption scanning radiography setup.

Scanning generally offers an inexpensive way for large area imaging due to the moderate costs of one-dimensional detectors. Even though our method needs at least four lines it will still be favorable because the required grating size and, therefore, the costs for fabrication can be reduced. The nonorthogonal angle of incidence onto the gratings can severely affect the phase contrast formation. By only using a small strip along the grating bars this problem can be overcome because nonorthogonality with respect to the direction of the grating bars does not affect it at all. Furthermore, the difficulties of alignment, homogeneity, and distortion of large gratings on their native wafers can be reduced. Finally, we conclude that it is feasible to implement this technique into many of the existing x-ray scanning systems because this technique neither demands for any microfocus tube nor is the radiation dose significantly increased.

\section{ACKMOWLEDGMENTS}

This work was supported by the Swiss Commission for Technology and Innovation KTI/CTI under Contract No. 7796.2 DCPP-NM. The authors gratefully acknowledge L. Tlustos from the Medipix2 collaboration for providing us with one detector module.

\footnotetext{
${ }^{1}$ U. Bonse and M. Hart, Appl. Phys. Lett. 6, 155 (1965).

${ }^{2}$ V. A. Somenkov, A. K. Tkalich, and S. S. Shilstein, Sov. Tech. Phys. Lett. 61, 197 (1991).

${ }^{3}$ V. V. Protopopov and J. Sobota, Opt. Commun. 213, 267 (2002).

${ }^{4}$ D. Chapman, W. Thomlinson, R. E. Johnston, D. Washburn, E. Pisano, N. Gmür, Z. Zhong, R. Menk, F. Arfelli, and D. Sayers, Phys. Med. Biol. 42, 2015 (1997).

${ }^{5}$ V. N. Ingal and E. A. Beliaevskaya, J. Phys. D 28, 2314 (1995).

${ }^{6}$ T. J. Davis, D. Gao, T. E. Gureyev, A. W. Stevenson, and S. W. Wilkins, Nature 373, 595 (1995).
} 
${ }^{7}$ S. W. Wilkins, T. E. Gureyev, D. Gao, A. Pogany, and A. W. Stevenson, Nature 384, 335 (1996).

${ }^{8}$ S. C. Mayo, T. J. Davis, T. E. Gureyev, P. R. Miller, D. Paganin, A. Pogany, A. W. Stevenson, and S. W. Wilkins, Opt. Express 11, 2289 (2003).

${ }^{9}$ K. A. Nugent, T. E. Gureyev, D. F. Cookson, D. Paganin, and Z. Barnea, Phys. Rev. Lett. 77, 2961 (1996).

${ }^{10}$ P. Cloetens, W. Ludwig, J. Baruchel, D. van Dyck, J. van Landuyt, J. P. Guigay, and M. Schlenker, Appl. Phys. Lett. 75, 2912 (1999).

${ }^{11}$ R. Fitzgerald, Phys. Today 53, 23 (2000).

${ }^{12}$ A. Momose, T. Takeda, Y. Itai, and K. Hirano, Nat. Med. 2, 473 (1996).

${ }^{13}$ A. Momose, Opt. Express 11, 2303 (2003).

${ }^{14}$ A. Momose, Jpn. J. Appl. Phys., Part 1 44, 6355 (2005).

${ }^{15}$ A. Momose, W. Yashiro, Y. Takeda, Y. Suzuki, and T. Hattori, Jpn. J. Appl. Phys., Part 1 45, 5254 (2006).

${ }^{16}$ J. Li, Z. Zhong, R. Lidtke, K. E. Kuettner, C. Peterfy, E. Aliyeva, and C. Muehleman, J. Anat. 202, 463 (2003).

${ }^{17}$ C. Muehleman, J. Li, Z. Zhong, J. G. Brankov, and M. N. Wernick, J. Anat. 208, 115 (2006).

${ }^{18}$ F. Pfeiffer, T. Weitkamp, O. Bunk, and C. David, Nature Physics 2, 258 (2006).

${ }^{19}$ F. Pfeiffer, C. Kottler, O. Bunk, and C. David, Phys. Rev. Lett. 98, 108105
(2007).

${ }^{20}$ C. Kottler, C. David, F. Pfeiffer, and O. Bunk, Opt. Express 15, 1175 (2007).

${ }^{21}$ C. David, E. Ziegler, and B. Nöhammer, J. Synchrotron Radiat. 8, 1054 (2001).

${ }^{22}$ C. David, J. Bruder, T. Rohbeck, C. Kottler, C. Grünzweig, A. Diaz, O. Bunk and F. Pfeiffer, Microelectron. Eng. (to be published).

${ }^{23}$ E. Wolf, Prog. Opt. 26, 349 (1988).

${ }^{24}$ T. Weitkamp, A. Diaz, C. David, F. Pfeiffer, M. Stampanoni, P. Cloetens, and E. Ziegler, Opt. Express 13, 6296 (2005).

${ }^{25}$ F. Pfeiffer, C. Grünzweig, O. Bunk, G. Frei, E. Lehmann, and C. David, Phys. Rev. Lett. 96, 215505 (2006).

${ }^{26}$ T. Weitkamp, B. Nöhammer, A. Diaz, C. David, and E. Ziegler, Appl. Phys. Lett. 86, 054101 (2005).

${ }^{27}$ T. Weitkamp, B. Nöhammer, A. Diaz, C. David, and E. Ziegler, Appl. Phys. Lett. 86, 119902 (2005).

${ }^{28}$ C. David, B. Nöhammer, H. H. Solak, and E. Ziegler, Appl. Phys. Lett. 81, 3287 (2002).

${ }^{29}$ X. Llopart, M. Campbell, R. Dinapoli, D. San Segundo, and E. Pernigotti, IEEE Trans. Nucl. Sci. 49, 2279 (2002).

${ }^{30}$ The periodicity $p_{2}$ needs to satisfy the condition $p_{2}=p_{1}[(l+d) / l]$ in order to match the periodicity of the interference pattern $I(x)$. 